The Agriculturists 12(2): 56-63 (2014) ISSN 2304-7321 (Online), ISSN 1729-5211 (Print)

A Scientific Journal of Krishi Foundation

Indexed Journal

\title{
Response of Okra (Abelmoschus esculentus L.) to Growth Regulators and Organic Manures
}

\author{
M. S. Chowdhury, Z. Hasan, K. Kabir, M. Shah Jahan and M. H. Kabir ${ }^{*}$ \\ Department of Horticulture, Sher-e-Bangla Agricultural University, Sher-e-Bangla Nagar, Dhaka, \\ Bangladesh \\ *Corresponding author and Email: kabirsau@yahoo.com
}

Received: 25 May 2014

Accepted: 13 December 2014

\begin{abstract}
A study was conducted at the Horticulture Farm, Sher-e-Bangla Agricultural University, Bangladesh during April to September, 2012 to determine the suitability of selected plant growth regulators and the proper use and effectiveness of selected organic manures and also their suitable combinations for successful okra production. The experiment consisted of two factors: factor A: growth regulators as $\mathrm{G}_{0}$ : control (water), $\mathrm{G}_{1}: \mathrm{GA}_{3}(100 \mathrm{ppm})$ and $\mathrm{G}_{2}$ : Miraculan $(1000 \mathrm{ppm})$ and factor $\mathrm{B}$ : organic manures as $-\mathrm{OM}_{0}$ : control (no manure), $\mathrm{OM}_{1}$ : vermicompost $\left(9 \mathrm{t} / \mathrm{ha}\right.$ ) and $\mathrm{OM}_{2}$ : poultry manure $(11.5 \mathrm{t} / \mathrm{ha})$. The combined use of $\mathrm{GA}_{3}$ and poultry manure produced the tallest plants. Both the growth regulators and organic manures enhanced early flowering. In case of growth hormone, the highest yield (16.67 t/ha) was recorded from $G_{1}$ followed by $G_{2}(16.49 \mathrm{t} / \mathrm{ha})$. The highest yield $(18.03 \mathrm{t} / \mathrm{ha})$ was found from $\mathrm{OM}_{2}$, closely followed by $\mathrm{OM}_{1}(17.59 \mathrm{t} / \mathrm{h})$. Considering the treatment combinations, the highest yield was harvested from $\mathrm{G}_{1} \mathrm{OM}_{2}(19.62 \mathrm{t} / \mathrm{ha})$, followed by $\mathrm{G}_{1} \mathrm{OM}_{1}(19.01 \mathrm{t} / \mathrm{h}), \mathrm{G}_{2} \mathrm{OM}_{1}(18.42 \mathrm{t} / \mathrm{h})$ and $\mathrm{G}_{2} \mathrm{OM}_{2}$ $(18.30 \mathrm{t} / \mathrm{h})$, respectively.
\end{abstract}

Keywords: Okra, $\mathrm{GA}_{3}$, Miraculan, vermicompost, poultry manure

\section{Introduction}

Okra or bhendi (Abelmoschus esculentus L.), belonging to the family Malvaceae, is an important vegetable crop of the world and is cultivated in Bangladesh in summer. It is a tropical and sub-tropical plant in which tender pods are used as a vegetable. These green fruits are rich sources of vitamins; calcium, potassium and other minerals. In Bangladesh, vegetable production is not uniform round the year: plenty in winter but less in summer. Around $30 \%$ of total vegetables are produced during summer and $70 \%$ in winter (Hossain, 1992). The present consumption of vegetables in Bangladesh is 112 g/day/capita (23 g leafy vegetables, 89 g non- leafy vegetables), which is far below the minimum average requirement of 400 g/day/capita (FAO/WHO, 2003). Therefore, there is a big gap between the requirement and the supply of vegetables in Bangladesh. Successful okra production may contribute partially in solving vegetable scarcity in summer. Okra production in the country is low compared to other countries. Total production of okra was about 240 thousand tons from 7287.5 ha in 2009 and the average yield was about $3.38 \mathrm{t} / \mathrm{ha}$ (BBS, 2010).

Plant growth regulators (PGR's) are organic compounds, which in small amounts modify physiological process of plants. PGR influences 
plant height, number of leaves, length of the internode, number of days for first flower initiation, fruit quality, number of fruits, fruit weight, and number of fruits per plant of okra (Kokare et al., 2006 and Nawalkar et al., 2007). Gibberellin (GA) plays an essential role in many aspects of plant growth and development, such as stem elongation and flowering (Yamaguchi and Kamiya, 2000). Miraculan (triacontanol $0.05 \%$ ) is also a PGR with effective metabolic activator used for enlarging fruit size, yield of different fruits and vegetable crops.

Organic manures such as farm yard manure, poultry manure, vermicompost etc. are very active and important for soil. It furnishes large portion of macro and micro nutrients, protects soil against erosion, supplies the cementing substance for desirable soil aggregate formation and loosen soil. Application of vermicompost and poultry manure subsequently increases yield attributes and yield of okra (Sameera et al., 2005). In addition, the product from organic manure is beneficial for health and the natural eco-system. Therefore, the present investigation was carried out to find out the suitability of selected plant growth regulators and the proper use and effectiveness of selected organic manures and also their suitable combinations for successful okra production in Bangladesh.

\section{Materials and Methods}

The study was conducted at the Horticulture Farm, Sher-e-Bangla Agricultural University, Dhaka, Bangladesh from April to September 2012, to determine the response of okra to growth hormone and organic manure. BARI Dherosh 1 was used as the test crop. The experiment consisted of two factors: factor A: growth regulators (three levels) as $-\mathrm{G}_{0}$ : control (no growth regulators), $\mathrm{G}_{1}$ : $\mathrm{GA}_{3}$ (gibrellic acid @ 100 ppm) and $\mathrm{G}_{2}$ : Miraculan @ 1000 ppm (Triacontanol $0.05 \%$ ) and the factor B: organic manure (three levels) as - $\mathrm{OM}_{0}$ : control (no manuring), $\mathrm{OM}_{1}$ : vermicompost $\left(9 \mathrm{t} \mathrm{ha}^{-1}\right)$ and $\mathrm{OM}_{2}$ : poultry manure $\left(11.5 \mathrm{t} \mathrm{ha}^{-1}\right)$. No chemical fertilizer was applied in the experiment.
The two factors experiment was laid out in a Randomized Complete Block Design (RCBD) with three replications. The total experimental plot $\left(114.75 \mathrm{~m}^{2}\right.$ with length $15.3 \mathrm{~m}$ and width 7.5 m) was divided into three equal blocks. Each block was then divided into 9 plots where 9 treatment combinations were allotted at random. Thus there were 27 unit plots.

Seeds were sown in the plots maintaining the distance of $50 \times 40 \mathrm{~cm}$. Intercultural operations were done as and when necessary. Five plants were selected for data collection. Data were recorded at 20, 40 and 60 days after sowing. The data recorded on different characteristics were plant height $(\mathrm{cm})$, number of leaves per plant, number of internodes per plant, fresh weight of plant $(\mathrm{g})$, dry matter of plant, days required for $50 \%$ flowering, number of flower buds per plant, number of pods per plant, pod length $(\mathrm{cm})$, pod diameter $(\mathrm{cm})$ and yield of okra. The mean values of all the recorded characteristics were evaluated and analysis of variance was performed using the ' $F$ ' (variance ratio) test. The significance of the difference among the treatment means was estimated by Duncan's Multiple Range Test (DMRT) (Gomez and Gomez, 1984).

\section{Results and Discussion}

\subsection{Plant height}

Plant height showed significant differences due to the influence of various growth hormones applied at 20, 40, and 60 days after sowing (DAS). The tallest plants $(14.82,72.46$ and 89.24 $\mathrm{cm})$ were recorded from $\mathrm{G}_{1}\left(\mathrm{GA}_{3} @ 100 \mathrm{ppm}\right)$ and at all the DAS, while the shortest plants $(12.93,64.00$ and $78.57 \mathrm{~cm})$ were measured from $\mathrm{G}_{0}$ (Figure 1). Singh et al. (1999) reported that $\mathrm{GA}_{3}$ increased plant height of okra. At 20, 40 and $60 \mathrm{DAS}$, the longest plants $(15.65,76.91$ and $89.68 \mathrm{~cm}$ ) were noted from $\mathrm{OM}_{2}$ (poultry manure@11.5 t ha ${ }^{-1}$ ) but the shortest plants $(11.99,57.99$ and $78.20 \mathrm{~cm})$ were found from $\mathrm{OM}_{0}$ as control at all the DAS (Figure 2). Pavan et al. (2004) reported that the poultry manure increased plant height over the untreated control. 
Vermicompost and poultry manure subsequently increased yield attributing characteristics of okra (Prakash and Bhadoria, 2004). The longest plants $(17.32,84.39$ and $96.20 \mathrm{~cm})$ were recorded from $\mathrm{G}_{1} \mathrm{OM}_{2}\left(\mathrm{GA}_{3} @ 100\right.$ ppm and poultry manure @ $11.5 \mathrm{t} \mathrm{ha}^{-1}$ ) at 20,40 and 60 DAS, respectively.

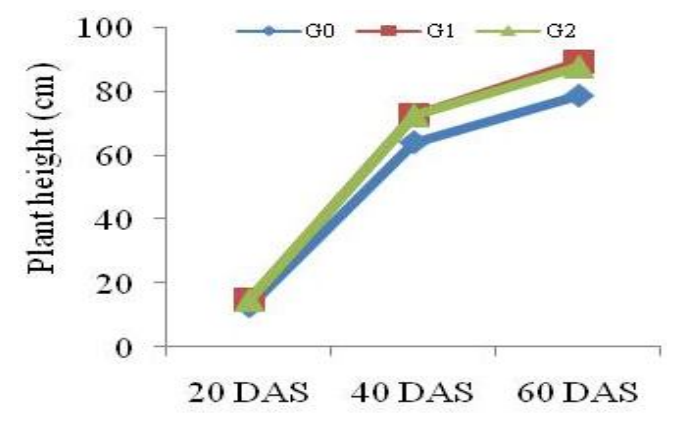

Days after sowing (DAS)
On the other hand, the shortest plants (11.47, 56.50 and $72.20 \mathrm{~cm}$ ) were recorded from $\mathrm{G}_{0} \mathrm{OM}_{0}$ (no growth hormone and no organic fertilizer) (Table 1). It was revealed that the combined use of $\mathrm{GA}_{3}$ and poultry manure @ $11.5 \mathrm{t} \mathrm{ha}^{-1}$ produced the tallest plant under that trial.

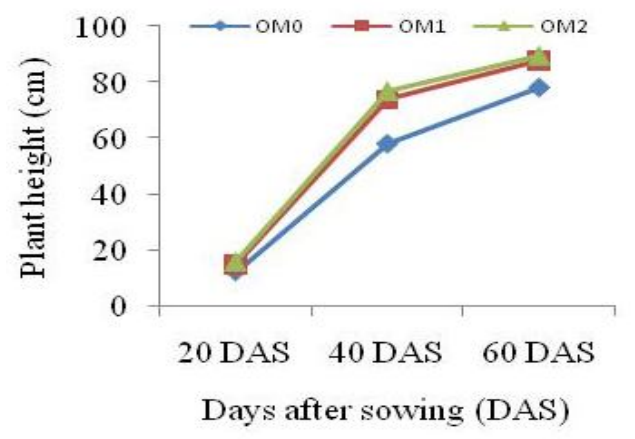

Figure 2. Effect of organic manures on the plant height at different davs after sowing of okra

Table 1. Effect of growth regulators and organic manures on number of leaves and number of internodes per plant of okra

\begin{tabular}{|c|c|c|c|c|c|c|}
\hline \multirow[t]{2}{*}{ Treatment } & \multicolumn{3}{|c|}{ Number of leaves per plant } & \multicolumn{3}{|c|}{ Number of internodes } \\
\hline & $20 \mathrm{DAS}$ & $40 \mathrm{DAS}$ & 60 DAS & $20 \mathrm{DAS}$ & 40 DAS & $60 \mathrm{DAS}$ \\
\hline \multicolumn{7}{|c|}{ Growth regulators } \\
\hline $\mathrm{G}_{0}$ & $5.66 \mathrm{~b}$ & $21.86 \mathrm{~b}$ & $43.07 \mathrm{~b}$ & $5.19 \mathrm{~b}$ & $12.94 \mathrm{~b}$ & $19.37 \mathrm{~b}$ \\
\hline $\mathrm{G}_{1}$ & $7.00 \mathrm{a}$ & $28.21 \mathrm{a}$ & $48.73 \mathrm{a}$ & $5.93 \mathrm{a}$ & $14.16 \mathrm{a}$ & $21.61 \mathrm{a}$ \\
\hline $\mathrm{G}_{2}$ & $6.94 \mathrm{a}$ & $25.90 \mathrm{a}$ & $48.71 \mathrm{a}$ & $5.80 \mathrm{a}$ & $14.11 \mathrm{a}$ & $21.22 \mathrm{a}$ \\
\hline $\operatorname{LSD}_{(0.05)}$ & 0.241 & 3.183 & 1.790 & 0.325 & 0.617 & 0.539 \\
\hline $\begin{array}{l}\text { Level of } \\
\text { significance }\end{array}$ & 0.01 & 0.01 & 0.01 & 0.01 & 0.01 & 0.01 \\
\hline \multicolumn{7}{|c|}{ Organic manures } \\
\hline $\mathrm{OM}_{0}$ & $5.67 \mathrm{~b}$ & $19.01 \mathrm{c}$ & $40.78 \mathrm{~b}$ & $4.87 \mathrm{c}$ & $12.09 \mathrm{~b}$ & $19.20 \mathrm{~b}$ \\
\hline $\mathrm{OM}_{1}$ & $6.88 \mathrm{a}$ & $26.64 b$ & $49.10 \mathrm{a}$ & $5.84 \mathrm{~b}$ & $14.33 \mathrm{a}$ & $21.32 \mathrm{a}$ \\
\hline $\mathrm{OM}_{2}$ & $7.06 \mathrm{a}$ & $30.31 \mathrm{a}$ & $50.63 \mathrm{a}$ & $6.21 \mathrm{a}$ & $14.79 \mathrm{a}$ & $21.68 \mathrm{a}$ \\
\hline $\operatorname{LSD}_{(0.05)}$ & 0.241 & 3.183 & 1.790 & 0.325 & 0.617 & 0.539 \\
\hline $\begin{array}{l}\text { Level of } \\
\text { significance }\end{array}$ & 0.01 & 0.01 & 0.01 & 0.01 & 0.01 & 0.01 \\
\hline $\mathrm{CV}(\%)$ & 7.69 & 12.58 & 6.82 & 5.78 & 4.49 & 6.60 \\
\hline
\end{tabular}

In a column the mean values having similar letter(s) are statistically similar and those having dissimilar letter(s) differ significantly at the 0.05 level of significance

$\mathrm{G}_{0}$ : Control (no growth regulators/water), $\mathrm{G}_{1}$ : GA 3 (Gibberellic acid @100 ppm), $\mathrm{G}_{2}$ : Miraculan @ $1000 \mathrm{ppm}$ (Triacontanol 0.05\%), $\mathrm{OM}_{0}$ : Control (no manure), $\mathrm{OM}_{1}$ : Vermicompost @9 $\mathrm{t} \mathrm{ha}^{-1}, \mathrm{OM}_{2}$ : Poultry Manure @ $11.5 \mathrm{t} \mathrm{ha}^{-1}$ 


\subsection{Number of leaves per plant}

The maximum numbers of leaves per plant (7.00, 28.21 and 48.73) were counted from $G_{1}$ and at all the DAS. Oppositely the minimum numbers of leaves per plant $(5.66,21.86$ and 43.07) were obtained from $\mathrm{G}_{0}$ (Table 1). Singh and Mahesh (2005) reported the highest number of leaves per plant with 75 ppm $\mathrm{GA}_{3}$. At 20, 40 and 60 DAS, the maximum numbers of leaves per plant (7.06, 30.31 and 50.63) were found from $\mathrm{OM}_{2}$, whereas the minimum numbers of leaves per plant (5.67, 19.01 and 40.78) were observed from $\mathrm{OM}_{0}$ (Table 1). The maximum numbers of leaves per plant $(7.67,34.93$ and 54.70) were obtained from $\mathrm{G}_{1} \mathrm{OM}_{2}$ at 20, 40 and 60 DAS, respectively. On the contrary, the minimum numbers of leaves per plant $(4.83,18.37$ and 41.10) were found from $\mathrm{G}_{0} \mathrm{OM}_{0}$, (Table 2).

\subsection{Number of internodes per plant}

Different growth hormones showed significant differences for number of internodes per plant at 20,40 , and 60 DAS. The maximum numbers of internodes per plant $(5.93,14.16$ and 21.61) were recorded from $G_{1}$ whereas at all the DAS, the minimum numbers of internodes $(5.19,12.94$ and 19.37) were recorded from $\mathrm{G}_{0}$ (Table 1). At 20, 40 and 60 DAS, the maximum numbers of internodes $(6.21,14.79$ and 21.68) were recorded from $\mathrm{OM}_{2}$. Oppositely, the minimum numbers of internodes (4.87, 12.09 and 19.20) were found from $\mathrm{OM}_{0}$ (Table 1). The maximum numbers of internodes $(6.87,15.87$ and 23.03) were recorded from $\mathrm{G}_{1} \mathrm{OM}_{2}$ at 20, 40 and $60 \mathrm{DAS}$, respectively whereas the minimum numbers of internodes (4.67, 11.87 and 18.27) were recorded from $\mathrm{G}_{0} \mathrm{OM}_{0}$, respectively (Table 2).

Table 2. Interaction effect of growth regulators and organic manures on plant height, number of leaves and number of internodes per plant of okra

\begin{tabular}{cccccccccc}
\hline Treatment & \multicolumn{3}{c}{ Plant height (cm) at } & \multicolumn{3}{c}{$\begin{array}{c}\text { Number of leaves per plant } \\
\text { at }\end{array}$} \\
\cline { 2 - 10 } & 20 DAS & 40 DAS & 60 DAS & 20 DAS & 40 DAS & 60 DAS & 20 DAS 40 DAS & 60 DAS \\
\hline $\mathrm{G}_{0} \mathrm{OM}_{0}$ & $11.47 \mathrm{ef}$ & $56.50 \mathrm{e}$ & $72.20 \mathrm{f}$ & $4.83 \mathrm{e}$ & $18.37 \mathrm{e}$ & $41.10 \mathrm{~cd}$ & $4.67 \mathrm{e}$ & $11.87 \mathrm{e}$ & $18.27 \mathrm{e}$ \\
$\mathrm{G}_{0} \mathrm{OM}_{1}$ & $13.11 \mathrm{de}$ & $65.79 \mathrm{~d}$ & $81.09 \mathrm{e}$ & $5.99 \mathrm{~cd}$ & $21.97 \mathrm{cde}$ & $42.70 \mathrm{bc}$ & $5.27 \mathrm{~d}$ & $13.20 \mathrm{~d}$ & $19.83 \mathrm{~d}$ \\
$\mathrm{G}_{0} \mathrm{OM}_{2}$ & $14.21 \mathrm{~cd}$ & $69.72 \mathrm{~cd}$ & $82.42 \mathrm{de}$ & $6.17 \mathrm{~cd}$ & $25.23 \mathrm{bcd}$ & $45.40 \mathrm{~b}$ & $5.63 \mathrm{~cd}$ & $13.77 \mathrm{~cd}$ & $20.00 \mathrm{~d}$ \\
$\mathrm{G}_{1} \mathrm{OM}_{0}$ & $10.79 \mathrm{f}$ & $51.67 \mathrm{e}$ & $77.75 \mathrm{ef}$ & $5.83 \mathrm{~d}$ & $18.63 \mathrm{e}$ & $38.70 \mathrm{~d}$ & $4.43 \mathrm{e}$ & $11.17 \mathrm{e}$ & $19.20 \mathrm{~d}$ \\
$\mathrm{G}_{1} \mathrm{OM}_{1}$ & $16.36 \mathrm{ab}$ & $80.37 \mathrm{ab}$ & $93.75 \mathrm{ab}$ & $7.49 \mathrm{ab}$ & $31.07 \mathrm{ab}$ & $52.80 \mathrm{a}$ & $6.50 \mathrm{ab}$ & $15.30 \mathrm{ab}$ & $22.60 \mathrm{ab}$ \\
$\mathrm{G}_{1} \mathrm{OM}_{2}$ & $17.32 \mathrm{a}$ & $84.39 \mathrm{a}$ & $96.20 \mathrm{a}$ & $7.67 \mathrm{a}$ & $34.93 \mathrm{a}$ & $54.70 \mathrm{a}$ & $6.87 \mathrm{a}$ & $15.87 \mathrm{a}$ & $23.03 \mathrm{a}$ \\
$\mathrm{G}_{2} \mathrm{OM}_{0}$ & $13.70 \mathrm{~cd}$ & $65.81 \mathrm{~d}$ & $84.64 \mathrm{cde}$ & $6.33 \mathrm{c}$ & $20.03 \mathrm{de}$ & $42.53 \mathrm{bc}$ & $5.50 \mathrm{~d}$ & $13.23 \mathrm{~d}$ & $20.13 \mathrm{~d}$ \\
$\mathrm{G}_{2} \mathrm{OM}_{1}$ & $14.27 \mathrm{~cd}$ & $74.93 \mathrm{bc}$ & $88.19 \mathrm{bcd}$ & $7.16 \mathrm{~b}$ & $26.90 \mathrm{bc}$ & $51.80 \mathrm{a}$ & $5.77 \mathrm{~cd}$ & $14.50 \mathrm{bc}$ & $21.53 \mathrm{c}$ \\
$\mathrm{G}_{2} \mathrm{OM}_{2}$ & $15.42 \mathrm{bc}$ & $76.64 \mathrm{abc}$ & $90.41 \mathrm{abc}$ & $7.33 \mathrm{ab}$ & $30.77 \mathrm{ab}$ & $51.80 \mathrm{a}$ & $6.13 \mathrm{bc}$ & $14.73 \mathrm{bc}$ & $22.00 \mathrm{bc}$ \\
\hline $\mathrm{LSD}_{(0.05)}$ & 1.684 & 7.516 & 6.481 & 0.417 & 5.514 & 3.100 & 0.564 & 1.068 & 0.934 \\
$\mathrm{Level} \mathrm{of}$ & 0.01 & 0.01 & 0.05 & 0.05 & 0.05 & 0.01 & 0.01 & 0.01 & 0.01 \\
significance & & & & & & & & & \\
$\mathrm{CV}(\%)$ & 6.91 & 6.24 & 4.40 & 7.69 & 12.58 & 6.82 & 5.78 & 4.49 & $6.60[[$ \\
\hline
\end{tabular}

In a column the mean values having similar letter(s) are statistically similar and those having dissimilar letter(s) differ significantly at the 0.05 level of significance

$\mathrm{G}_{0}$ : Control (no growth regulators/water), $\mathrm{G}_{1}: \mathrm{GA}_{3}$ (Gibberellic acid @ $100 \mathrm{ppm}$ ), $\mathrm{G}_{2}$ : Miraculan @ $1000 \mathrm{ppm}$ (Triacontanol $0.05 \%$ ), $\mathrm{OM}_{0}$ : Control (no manure), $\mathrm{OM}_{1}$ : Vermicompost @9 $\mathrm{t} \mathrm{ha}^{-1}, \mathrm{OM}_{2}$ : Poultry Manure @ $11.5 \mathrm{t} \mathrm{ha}^{-1}$ 


\subsection{Fresh weight of plant}

The highest fresh weight of plant $(84.70 \mathrm{~g})$ was obtained from $G_{1}$ which was statistically similar (82.93) to that form $G_{2}$, whereas the lowest weight (74.38) was found from $\mathrm{G}_{0}$ (Table 3). The highest fresh weight of plant (88.28) was recorded from $\mathrm{OM}_{2}$ whereas, the lowest (69.93) was found from $\mathrm{OM}_{0}$ (Table 3). The highest fresh weight of plant (97.00) was recorded from $\mathrm{G}_{1} \mathrm{OM}_{2}$, whereas the lowest weight $(65.99 \mathrm{~g})$ was recorded from $\mathrm{G}_{0} \mathrm{OM}_{0}$ (Table 4).

\subsection{Dry weight of plant}

The highest dry weight of plant (11.09 g) was found from $G_{1}$, again the lowest (9.53) was obtained from $\mathrm{G}_{0}$ (Table 3). Vijayaraghavan (1999) reported that $50 \mathrm{ppm}$ gibberellic acid produced the maximum total dry matter than the control. The highest dry weight of plant (11.45) was observed from $\mathrm{OM}_{2}$, while the lowest (8.98) found from $\mathrm{OM}_{0}$ (Table 3). The highest dry weight of plant (12.54) was obtained from $\mathrm{G}_{1} \mathrm{OM}_{2}$, but the lowest (8.43 g) was recorded from $\mathrm{G}_{0} \mathrm{OM}_{0}$ (Table 4).

\subsection{Days to $50 \%$ flowering}

The highest days required for $50 \%$ flowering (47.11) was recorded from $\mathrm{G}_{0}$, while the lowest (43.22) was found from $\mathrm{G}_{1}$ (Table 3). Katung et al. (2007) reported that $\mathrm{GA}_{3}$ influenced the number of days to $50 \%$ flowering. The highest days required for $50 \%$ flowering (47.44) was obtained from $\mathrm{OM}_{0}$ while, the lowest (43.22) was recorded from $\mathrm{OM}_{1}$ (Table 3). The highest days required for $50 \%$ flowering (50.00) was obtained from $\mathrm{G}_{0} \mathrm{OM}_{0}$, again the lowest (40.00) was found from $\mathrm{G}_{2} \mathrm{OM}_{1}$ (Table 4).

\subsection{Number of flower buds per plant}

The maximum number of flower buds per plant (43.86) was recorded from $\mathrm{G}_{2}$ which was statistically similar with $\mathrm{G}_{1}$ (42.98). On the other hand, the minimum (36.18) was recorded from $\mathrm{G}_{0}$ (Table 3). The maximum number of flower buds (43.13) was observed from $\mathrm{OM}_{1}$ which was similar to $\mathrm{OM}_{2}$ (42.66), while the minimum
(37.22) was obtained from $\mathrm{OM}_{0}$ (Table 3). The maximum number of flower buds (45.53) was observed from $\mathrm{G}_{1} \mathrm{OM}_{2}$. On the other hand, the minimum (33.43) was found from $\mathrm{G}_{0} \mathrm{OM}_{0}$ (Table 4).

\subsection{Number of pods per plant}

Significant variation was recorded for the number of pods per plant due to the application of different growth hormones. The maximum number of pods per plant (33.77) was recorded from $\mathrm{G}_{1}$ whereas the minimum (24.30) was observed from $\mathrm{G}_{0}$ (Table 3). Vijayaraghavan (1999) reported that $50 \mathrm{ppm}$ gibberellic acid produced the highest number of fruits per plant. The maximum number of pods per plant (33.87) was found from $\mathrm{OM}_{2}$, whereas the minimum (23.12) was recorded from $\mathrm{OM}_{0}$ (Table 3). The maximum numbers of pods per plant (39.60) were found from $\mathrm{G}_{1} \mathrm{OM}_{2}$, and again the minimum (20.70) were observed from $\mathrm{G}_{0} \mathrm{OM}_{0}$ (Table 4).

\subsection{Pod length}

The longest pods $(17.66 \mathrm{~cm})$ were observed in $\mathrm{G}_{1}$ treated crops and the shortest pods $(15.33 \mathrm{~cm})$ were found from $\mathrm{G}_{0}$ (Table 3 ). The longest pods (17.72) were observed from $\mathrm{OM}_{2}$ whereas the shortest pods (15.67) were recorded from $\mathrm{OM}_{0}$ (Table 3). The longest pods (18.67) were recorded from $\mathrm{G}_{1} \mathrm{OM}_{2}$, while the shortest ones $(13.83 \mathrm{~cm})$ were recorded from $\mathrm{G}_{0} \mathrm{OM}_{0}$ (Table 4).

\subsection{Pod diameter}

Significant variation was recorded in pod diameter as an effect of growth hormones. The highest pod diameter $(1.77 \mathrm{~cm})$ was recorded from $\mathrm{G}_{1}$ whereas the lowest diameter (1.33) was recorded from $\mathrm{G}_{0}$ (Table 3). The highest pod diameter (1.84) was recorded from $\mathrm{OM}_{2}$ whereas the lowest diameter (1.22) was found from $\mathrm{OM}_{0}$ (Table 3). The highest pod diameter (2.11) was recorded from $\mathrm{G}_{1} \mathrm{OM}_{2}$ whereas the lowest pod diameter $(1.04 \mathrm{~cm})$ was obtained from $\mathrm{G}_{0} \mathrm{OM}_{0}$ (Table 4). 
Table 3. Effect of growth hormones and organic manures on the fresh weight, dry weight, yield contributing traits and yield of okra

\begin{tabular}{|c|c|c|c|c|c|c|c|c|}
\hline Treatment & $\begin{array}{l}\text { Fresh } \\
\text { weight } \\
\text { per plant } \\
\text { (g) }\end{array}$ & $\begin{array}{c}\text { Dry } \\
\text { weight } \\
\text { per plant } \\
(\mathrm{g})\end{array}$ & $\begin{array}{c}\text { Days } \\
\text { required } \\
\text { for } 50 \% \\
\text { flowering }\end{array}$ & $\begin{array}{c}\text { Flower } \\
\text { buds per } \\
\text { plant } \\
\text { (No.) }\end{array}$ & $\begin{array}{c}\text { Pods } \\
\text { per plant } \\
(\text { No. })\end{array}$ & $\begin{array}{c}\text { Pod } \\
\text { length } \\
(\mathrm{cm})\end{array}$ & $\begin{array}{c}\text { Pod } \\
\text { diameter } \\
(\mathrm{cm})\end{array}$ & $\begin{array}{l}\text { Yield } \\
\text { (t/ha) }\end{array}$ \\
\hline \multicolumn{9}{|c|}{ Growth regulators } \\
\hline $\mathrm{G}_{0}$ & $74.38 \mathrm{~b}$ & $9.53 \mathrm{~b}$ & $47.11 \mathrm{a}$ & $36.18 \mathrm{~b}$ & $24.30 \mathrm{c}$ & $15.33 \mathrm{~b}$ & $1.33 \mathrm{~b}$ & $14.08 \mathrm{~b}$ \\
\hline $\mathrm{G}_{1}$ & $84.70 \mathrm{a}$ & $11.09 \mathrm{a}$ & $43.22 \mathrm{~b}$ & $42.98 \mathrm{a}$ & $33.77 \mathrm{a}$ & $17.66 \mathrm{a}$ & $1.77 \mathrm{a}$ & $16.67 \mathrm{a}$ \\
\hline $\mathrm{G}_{2}$ & $82.93 \mathrm{a}$ & $10.79 \mathrm{a}$ & $43.89 \mathrm{~b}$ & $43.86 \mathrm{a}$ & $31.57 \mathrm{~b}$ & $17.61 \mathrm{a}$ & $1.70 \mathrm{a}$ & $16.49 \mathrm{a}$ \\
\hline $\operatorname{LSD}_{(0.05)}$ & 4.180 & 0.548 & 2.283 & 1.139 & 1.870 & 0.697 & 0.089 & 0.895 \\
\hline $\begin{array}{l}\text { Level of } \\
\text { significance }\end{array}$ & 0.01 & 0.01 & 0.01 & 0.01 & 0.01 & 0.01 & 0.01 & 0.01 \\
\hline \multicolumn{9}{|c|}{ Organic manures } \\
\hline $\mathrm{OM}_{0}$ & $69.93 \mathrm{c}$ & $8.98 \mathrm{~b}$ & $47.44 \mathrm{a}$ & $37.22 \mathrm{~b}$ & $23.12 b$ & $15.67 \mathrm{~b}$ & $1.22 \mathrm{c}$ & $11.62 \mathrm{~b}$ \\
\hline $\mathrm{OM}_{1}$ & $83.80 \mathrm{~b}$ & $10.99 \mathrm{a}$ & $43.22 \mathrm{~b}$ & $43.13 \mathrm{a}$ & $32.64 \mathrm{a}$ & $17.22 \mathrm{a}$ & $1.74 \mathrm{~b}$ & $17.59 \mathrm{a}$ \\
\hline $\mathrm{OM}_{2}$ & $88.28 \mathrm{a}$ & $11.45 \mathrm{a}$ & $43.56 \mathrm{~b}$ & $42.66 \mathrm{a}$ & $33.87 \mathrm{a}$ & $17.72 \mathrm{a}$ & $1.84 \mathrm{a}$ & $18.03 \mathrm{a}$ \\
\hline $\operatorname{LSD}_{(0.05)}$ & 4.180 & 0.548 & 2.283 & 1.139 & 1.870 & 0.697 & 0.089 & 0.895 \\
\hline $\begin{array}{l}\text { Level of } \\
\text { significance }\end{array}$ & 0.01 & 0.01 & 0.01 & 0.01 & 0.01 & 0.01 & 0.01 & 0.01 \\
\hline $\mathrm{CV}(\%)$ & 5.19 & 5.24 & 5.11 & 7.78 & 6.26 & 4.14 & 5.46 & 5.69 \\
\hline
\end{tabular}

In a column the mean values having similar letter(s) are statistically similar and those having dissimilar letter(s) differ significantly at the 0.05 level of significance

Table 4. Interaction effect of growth regulators and organic manures on fresh weight, dry weight, yield contributing characteristics and yield of okra

\begin{tabular}{|c|c|c|c|c|c|c|c|c|}
\hline Treatment & $\begin{array}{c}\text { Fresh } \\
\text { weight per } \\
\text { plant } \\
(\mathrm{g})\end{array}$ & $\begin{array}{c}\text { Dry } \\
\text { weight } \\
\text { per plant } \\
(\mathrm{g})\end{array}$ & $\begin{array}{c}\text { Days } \\
\text { required } \\
\text { for } 50 \% \\
\text { flowering }\end{array}$ & $\begin{array}{c}\text { Flower } \\
\text { buds per } \\
\text { plant } \\
\text { (No.) }\end{array}$ & $\begin{array}{c}\text { Pods } \\
\text { per plant } \\
\text { (No.) }\end{array}$ & $\begin{array}{c}\text { Pod } \\
\text { length } \\
(\mathrm{cm})\end{array}$ & $\begin{array}{c}\text { Pod } \\
\text { diameter } \\
(\mathrm{cm})\end{array}$ & $\begin{array}{l}\text { Yield } \\
\text { (t/ha) }\end{array}$ \\
\hline $\mathrm{G}_{0} \mathrm{OM}_{0}$ & $65.99 \mathrm{e}$ & $8.43 \mathrm{e}$ & $50.00 \mathrm{a}$ & $33.43 \mathrm{~d}$ & $20.70 \mathrm{e}$ & $13.83 \mathrm{~d}$ & $1.04 \mathrm{e}$ & $10.73 \mathrm{~d}$ \\
\hline $\mathrm{G}_{0} \mathrm{OM}_{1}$ & $76.39 \mathrm{~d}$ & $9.80 \mathrm{~d}$ & $45.67 \mathrm{~b}$ & $38.07 \mathrm{c}$ & $25.97 \mathrm{~d}$ & $15.99 \mathrm{c}$ & $1.43 \mathrm{~d}$ & $15.33 b$ \\
\hline $\mathrm{G}_{0} \mathrm{OM}_{2}$ & $80.76 \mathrm{~cd}$ & $10.37 \mathrm{~cd}$ & $45.67 \mathrm{~b}$ & $37.03 \mathrm{c}$ & $26.23 \mathrm{~d}$ & $16.17 \mathrm{c}$ & $1.54 \mathrm{~d}$ & $16.17 \mathrm{~b}$ \\
\hline $\mathrm{G}_{1} \mathrm{OM}_{0}$ & $64.80 \mathrm{e}$ & $8.77 \mathrm{e}$ & $41.67 \mathrm{bc}$ & $38.07 \mathrm{c}$ & $23.63 \mathrm{de}$ & $16.83 \mathrm{bc}$ & $1.18 \mathrm{e}$ & $11.37 \mathrm{~cd}$ \\
\hline $\mathrm{G}_{1} \mathrm{OM}_{1}$ & $92.31 \mathrm{ab}$ & $11.96 \mathrm{ab}$ & $44.00 \mathrm{bc}$ & $45.33 \mathrm{a}$ & $38.07 \mathrm{ab}$ & $17.49 \mathrm{ab}$ & $2.01 \mathrm{ab}$ & $19.01 \mathrm{a}$ \\
\hline $\mathrm{G}_{1} \mathrm{OM}_{2}$ & $97.00 \mathrm{a}$ & $12.54 \mathrm{a}$ & $44.00 \mathrm{bc}$ & $45.53 \mathrm{a}$ & $39.60 \mathrm{a}$ & $18.67 \mathrm{a}$ & $2.11 \mathrm{a}$ & $19.62 \mathrm{a}$ \\
\hline $\mathrm{G}_{2} \mathrm{OM}_{0}$ & $79.00 \mathrm{~d}$ & $9.73 \mathrm{~d}$ & $50.67 \mathrm{a}$ & $40.17 \mathrm{~b}$ & $25.03 \mathrm{~d}$ & $16.33 \mathrm{bc}$ & $1.43 \mathrm{~d}$ & $12.75 \mathrm{c}$ \\
\hline $\mathrm{G}_{2} \mathrm{OM}_{1}$ & $82.71 \mathrm{~cd}$ & $11.21 \mathrm{bc}$ & $40.00 \mathrm{c}$ & $46.00 \mathrm{a}$ & $33.90 \mathrm{c}$ & $18.17 \mathrm{a}$ & $1.79 \mathrm{c}$ & $18.42 \mathrm{a}$ \\
\hline $\mathrm{G}_{2} \mathrm{OM}_{2}$ & 87.08 bc & $11.44 \mathrm{~b}$ & $41.00 \mathrm{c}$ & $45.40 \mathrm{a}$ & $35.77 \mathrm{bc}$ & $18.33 \mathrm{a}$ & $1.87 \mathrm{bc}$ & $18.30 \mathrm{a}$ \\
\hline $\operatorname{LSD}_{(0.05)}$ & 7.241 & 0.950 & 3.954 & 1.973 & 3.239 & 1.208 & 0.155 & 1.550 \\
\hline $\begin{array}{l}\text { Level of } \\
\text { significance }\end{array}$ & 0.01 & 0.05 & 0.01 & 0.05 & 0.01 & 0.05 & 0.01 & 0.05 \\
\hline $\mathrm{CV}(\%)$ & 5.19 & 5.24 & 5.11 & 7.78 & 6.26 & 4.14 & 5.46 & 5.69 \\
\hline
\end{tabular}

In a column the mean values having similar letter(s) are statistically similar and those having dissimilar letter(s) differ significantly at the 0.05 level of significance.

$\mathrm{G}_{0}$ : Control (no growth regulators/water), $\mathrm{G}_{1}$ : $\mathrm{GA}_{3}$ (Gibberellic acid @100 ppm), $\mathrm{G}_{2}$ : Miraculan @ 1000 ppm (Triacontanol 0.05\%), $\mathrm{OM}_{0}$ : Control (no manure), $\mathrm{OM}_{1}$ : Vermicompost @9 $\mathrm{t} \mathrm{ha}^{-1}, \mathrm{OM}_{2}$ : Poultry Manure @ $11.5 \mathrm{t} \mathrm{ha}^{-1}$ 


\subsection{Pod yield}

Different growth hormones induced significant variations in pod yield per hectare. The highest yield $(16.67 \mathrm{t})$ was observed from $\mathrm{G}_{1}$ whereas the lowest (14.08) was attained from $\mathrm{G}_{0}$ (Table 3). The results is in agreement with the findings of Vijayaraghavan (1999) who reported that 50 ppm gibberellic acid produced the highest fruit yield of $15.7 \mathrm{t} / \mathrm{ha}$ and the control yield was 8.07 t/ha. Surendra et al. (2006) also reported that $\mathrm{GA}_{3} @ 25$ and $50 \mathrm{ppm}$ gave the highest fruit yields (15.81 and 18.69 t/ha, respectively). The highest yield (18.03) was recorded from $\mathrm{OM}_{2}$, while the lowest (11.62) was found in $\mathrm{OM}_{0}$ (Table 3). Ushakumari et al. (1999) reported that vermicompost applied as an organic source @12 $\mathrm{t} / \mathrm{ha}+$ the full recommended dose of inorganic fertilizers resulted the highest yield (5.66 t/ha). Pavan et al. (2004) reported that involving 50\% $\mathrm{N}$ as urea $+50 \% \mathrm{~N}$ as poultry manure recorded the highest yield $(90.61 \mathrm{q} / \mathrm{ha})$. The highest yield (19.62) was recorded from $\mathrm{G}_{1} \mathrm{OM}_{2}$, while the lowest yield per hectare $(10.73 \mathrm{t})$ was observed from $\mathrm{G}_{0} \mathrm{OM}_{0}$ (Table 4).

\section{Conclusions}

From the findings of the present study, it is revealed that okra plants responded well to growth regulators $\left(\mathrm{GA}_{3}\right.$ and Miraculan) and organic manures (vermicompost and poultry manure). Therefore, farmers may use any one of the growth regulators along with vermicompost or poultry manure to increase the okra yield.

\section{References}

FAO/WHO. 2003. Food and Agriculture Organization of the United Nations. Diet, nutrition and the prevention of chronic diseases. Report of a Joint FAO/WHO Expert Consultation. WHO Technical Report Series 916. Geneva, World Health Organization.

Gomez, K. A. and Gomez, A. A. 1984. Statistical Procedure for Agricultural
Research, $2^{\text {nd }}$ end., John Wiley and Sons, Singapore, 28-192 pp.

Hossain, S. M. 1992. Status, constrains and strategies of vegetable research. In: Katherine and Eli. L (eds.). Vegetable production and marketing. Proceedings of a national review and planning Workshop held at BARI, Gazipur, Bangladesh. 26-29 January. 1992, 31 p.

Katung, M. D., Olarewaju, J. D. and Mohammed, S. G. 2007. Seasonal response of okra (Abelmoschus esculentus L.) varieties to gibberellic acid. Advances in Horticultural Science, 21(1): 14-18.

Kokare, R. T., Bhalerao, R. K., Prabu, T., Chavan, S. K., Bansode, A. B. and Kachare, G. S. 2006. Effect of plant growth regulators on growth, yield and quality of okra (Abelmoschusesculentus L.). Agricultural Science Digest, 26(3): 178-181.

Monthly Bulletin, BBS, February. 2010. Monthly Statistical Bulletin of Bangladesh. Bangladesh Bureau of Statistics, Ministry of Planning. Government of the People's Republic of Bangladesh, Dhaka, 44 p.

Nawalkar, L. R., Khiratkar, S. D., Badge, S. A., Chopde, N. K. and Dadgal, S. S. 2007. Effect of biofertilizers and growth regulator with reduced doses of NPK on growth and yield of okra. Journal of Soils and Crops, 17(1): 145-149.

Pavan, Y., Singh, P., Yadav, R. L. and Ram, L. 2004. Organic manures and nitrogen levels on okra cv. Varsha Uphar. Haryana Journal of Horticultural Sciences, 33(1/2): 124-126.

Prakash, Y. S. and Bhadoria, P. B. S. 2004. Relative efficacy of different organic manures on the performance of okra (Abelmoschus esculentus L.) grown in Lateritic soil. Tropical Agriculture, 81(4): 197-203. 
Sameera, D. L., Shankaraiah, V. and Srihari, D. 2005. Effect of packaging and storage on organic manures grown okra (Abelmoschus esculentus L. Moench). Journal of Research ANGRAU, 33(4): 3035.

Singh, R. K. and Mahesh, K. 2005. Response of summer season okra to plant growth regulators and foliar application of nitrogen. Haryana Journal of Horticultural Sciences, 34(1/2): 187-188.

Singh, R. K., Singh, G. P. and Singh, V. K. 1999. Effect of plant growth regulators and green fruit pickings on the seed production of Bhindi (Abelmoschus esculentus L.). Journal of Applied Biology, 9(1): 31-34.

Surendra, P., Nawalagatti, C. M., Chetti, M. B. and Hiremath, S. M. 2006. Effect of plant yield and yield components in okra. Karnataka Journal of Agricultural Sciences, 19(2): 264-267.

Ushakumari, K., Prabhakumari, P. and Padmaja, P. 1999. Efficiency of vermicompost on growth and yield of summer crop okra (Abelmoschus esculentus Moench). Journal of Tropical Agriculture, 37(1/2): 87-88.

Vijayaraghavan, H. 1999. Effect of seed treatment with plant growth regulators on bhendi (Abelmoschus esculentus L.,) grown under sodic soil conditions. Madras Agricultural Journal, 86(4/6): 247-249.

Yamaguchi, S. and Kamiya, Y. 2000. Gibberellin biosynthesis: its regulation by endogenous and environmental signals. Plant Cell Physiology, 41(3): 251-257. 